\title{
LA THÉORIE AUTRICHIENNE DES CYCLES: UNE THÉORIE DE LA RÉCURRENCE DES ERREURS COLLECTIVES D'ANTICIPATION
}

\author{
FRANÇOIS FACCHINI*
}

Resume: Cet article présente les développements récents de la théorie autrichienne des cycles. II se concentre sur les apports théoriques et soutient que désormais la théorie autrichienne des cycles est une théorie plurielle de la récurrence des erreurs collectives d'anticipation. Les économistes autrichiens s'accordent pour penser que la nationalisation de la monnaie est à l'origine de l'excès d'offre de monnaie qui crée une distorsion de la structure des taux d'intérêt des prêts et induit la phase de récession. Ils s'entendent aussi sur les raisons de la récurrence des erreurs d'anticipation et sur leur uniformité. La théorie des droits de propriété explique la récurrence des erreurs d'anticipation par la socialisation des risques. La théorie des anticipations explique les erreurs collectives par la centralisation des anticipations autour des décisions de la banque centrale et rend compte ainsi de l'instabilité des systèmes économiques. Les économistes autrichiens se divisent, en revanche, sur les raisons de cet excès d'offre de monnaie. II y a ceux qui soutiennent que cet excès d'offre se mesure par rapport à l'épargne monétaire et s'explique par la pratique des réserves fractionnaires (école de la libre circulation). II y a ceux, au contraire, qui estiment que cet excès doit être mesuré par rapport à la demande de monnaie et expliqué parl'absence de concurrence entre les monnaies (école de la banque libre).

* EDJ, Université de Reims Champagne Ardenne, Faculté de sciences économiques et de gestion, 57 bis rue Pierre Taittinger 51000 Reims, et Chercheur associé au LAEP, Université de Paris 1 Panthéon - Sorbonne, http://laep.univ-paris1.fr/ facchini/index.htm et facchini@univ-paris1.fr.

Cet article a été publié initialement dans l'Actualité Economique, numéro de Septembre 2004. Nous tenons à remercier son Comité de rédaction pour avoir accepté sa ré-édition. 
Mots clés: cycles, anticipations, droits de propriété, banque centrale, banque libre et erreur

Abstract: This paper offers an account of the recent development of Austrian Trade Cycle Theory. It focus on the theoretical contributions and argues that the Austrian's explanation of Trade Cycle is a theory of collective errors of expectations and of their recurrences. Austrian economists agree to explain the errors of individuals by the nationalization of money because it leads to an excess of money supply. Nevertheless, they disagree about the cause of this excess. Two explanations have been suggested. The first one measures the excess in relation with the demand of money. The second one evaluates the excess in relation with monetary saving. They agree, on the contrary, on the reasons of the recurrence of expectation errors and their uniformity. The theory of property rights explains the recurrence of expectation errors by the socialization of risk. The expectation theory explains the collective errors by the centralisation of expectations (big player hypothesis) on the central bank decisions. Therefore, the centralization of expectations explains the instability of market process.

Keywords: cycle, expectations, property rights, central banking, free banking and error

Classification JEL: E32, E58,

\section{INTRODUCTION}

La théorie autrichienne des cycles s'est développée au début du vingtième siècle avec les travaux de Ludwig von Mises $(1912,1966)$ et de Friedrich Hayek $(1931,1932)^{1}$. Elle s'est ensuite précisée avec les travaux de Murray Rothbard (1970, 1975), Ludwig Lachmann (1978), Roger Garrisson (1984, 1989) et/ou Gerald O'Driscoll et Mario Rizzo (1985). Il existe entre ces auteurs des différences et aucune version canonique de la théorie autrichienne des cycles ne s'est aujourd'hui imposée (Garrisson 1989, p.3). Tous s'accordent,

${ }^{1}$ Voir 1'article de Dostaler G. (2001). 
cependant, sur l'importance des liens entre les prix relatifs et la coordination inter-temporelle, autrement dit, la structure du capital pour expliquer les cycles. Tous restent fidèles de surcroît à l'idée que les cycles économiques ont une raison monétaire qui trouve son origine dans la fixation d'un taux d'intérêt des prêts inadaptée aux préférences inter-temporelles des individus sur le marché (Sechrest 1997, p.10).

Cette théorie des cycles a pourtant fait l'objet de plusieurs types de critiques. Dès les années trente, de nombreux économistes doutaient de sa capacité à rendre compte des faits. Plus tard, Axel Leijonhufvud (1986, p.417) a partagé cette opinion et soutenu que la théorie autrichienne des cycles avait été falsifiée parce que l'inflation ne produisait aucun boom de sur-investissement et que la stagflation des années 1970 permettait de constater l'inflation, mais ne permettait pas d'observer l'accélération de l'accumulation du capital et l'épargne forcée pourtant prévues par les économistes autrichiens. La théorie du capital, qui fonde la théorie autrichienne des cycles a, de plus, été jugée incorrecte ${ }^{2}$. L'une des conséquences majeures de ce constat serait qu'il n'est pas nécessaire de savoir si la demande de capital fluctue pour expliquer les cycles (Yeager 1986, p.382). Il suffirait d'expliquer le processus d'ajustement entre le taux d'intérêt et le changement des prix relatifs pour rendre compte des cycles. L'existence d'un déséquilibre monétaire expliquerait à lui seul les cycles (Yeager 1986 et Friedman 1993, p.172).

Malgré la virulence des critiques, les économistes autrichiens continuent de penser que les cycles s'expliquent par un excès d'offre de monnaie. Ils contestent, tout d'abord, la thèse selon laquelle la théorie autrichienne serait incapable de rendre compte de l'Histoire ${ }^{3}$ et des grandes crises ${ }^{4}$. Ils acceptent, en

\footnotetext{
2 Voir M. Blaug (1981, p.586).

${ }^{3}$ La crise allemande des années 20 a été expliquée par Bresciani-Turroni (1937). Le Roux et Levin (1998) montrent que l'explication autrichienne des crises s'applique
} 
revanche, d'abandonner la recherche d'une mesure objective du détour de production pour adopter une théorie radicalement subjective du capital. On peut alors estimer qu'ils renouvellent leur théorie en définissant les cycles comme le résultat d'un processus récurrent d'erreurs collectives d'anticipation.

Cet article se propose de présenter ce renouvellement de la théorie autrichienne des cycles. Il rappelle, tout d'abord, les enchaînements de la théorie autrichienne des cycles et l'importance qu'elle accorde à la coordination inter-temporelle des actions humaines sur le marché par les taux d'intérêt (2).

Il explique, ensuite, pourquoi l'institutionnalisation d'un système monétaire hiérarchisé autour d'une banque centrale suscite l'apparition d'une structure des taux d'intérêt des prêts qui ne reflète pas les préférences temporelles des agents. Il présente à cette occasion le débat entre l'école de la libre circulation et l'école de la banque libre. Ces deux écoles soutiennent que la nationalisation de la monnaie est à l'origine de l'instabilité des économies de marché, mais se divisent sur la manière dont ce changement institutionnel modifie la structure des taux d'intérêt des prêts. L'école de la banque libre estime que la structure des taux d'intérêt des prêts ne reflète pas les préférences temporelles parce que le marché de la monnaie n'est pas concurrentiel. L'école de la libre circulation soutient que c'est la généralisation de la pratique des réserves fractionnaires qui explique la distorsion de la structure des taux d'intérêt.

Il aborde, enfin les questions de la récurrence et de l'uniformité des erreurs (4). Pourquoi les entrepreneurs n'apprennent rien de leurs erreurs passées? Pourquoi font-ils

à la récession de l'économie sud-africaine de 1980-1996. Hughes (1997) explique la récession de 1990. Keeler (2001) montre la pertinence du modèle autrichien pour comprendre les cycles de l'économie américaine. Wainhouse (1984) met en perspective quelques évidences qui valident la théorie autrichienne des cycles.

4 Rothbard (1963) et Robbins (1934) analysent la grande crise de 1929 comme une illustration de la théorie autrichienne des cycles. Voir aussi les travaux de Skousen $(1988,1994)$. 
tous la même erreur au même moment? On rend compte de la récurrence des erreurs d'anticipation par la socialisation des risques. La nationalisation de la monnaie a les mêmes effets que la socialisation en général. Elle favorise le développement de comportements de passager clandestin (4.1). On explique l'uniformité des anticipations par le processus de convergence des anticipations autour des décisions de la banque centrale que provoque la centralisation du système monétaire. C'est parce que la banque centrale a les caractéristiques d'un «big player» qu'elle centralise le jeu des anticipations anéantissant ainsi leur diversité et, du même coup, les conditions de stabilité de l'économie de marché (4.2).

\section{LA THÉORIE AUTRICHIENNE DES CYCLES EST UNE THÉORIE DE LA MAUVAISE COORDINATION DES MARCHÉS DANS LE TEMPS}

Le cycle dans les théories de Mises et Hayek s'explique par une baisse du taux d'intérêt des prêts qui n'est pas provoquée par une hausse de l'épargne, mais par une décision discrétionnaire des banques.

\section{Taux d'intérêt des prêts et taux d'intérêt originaires}

L'évolution des taux d'intérêt des prêts reflète normalement l'évolution des taux d'intérêt originaires. Le taux d'intérêt originaire est «le rapport entre la valeur attribuée à la satisfaction dans le futur immédiat, et la valeur attribuée à la satisfaction du même besoin dans des temps plus éloignés. Il se manifeste en économie de marché par la dépréciation des biens à venir, comparés aux biens présents. C'est un rapport entre des prix de denrées, non un prix en lui-même» (Mises 1985, p.552 ou 1966, p.526). L'individu estime 
qu'une pomme aujourd'hui vaut 1 euro et qu'une pomme demain vaut 0,5 euro. Le rapport des prix entre biens présents et biens futurs est de deux. Le bien présent a deux fois plus de valeur que le bien futur. L'intérêt est donc une catégorie de l'action humaine.

L'abstinence, ainsi expliquée par la préférence pour le futur, rend possible l'échange différé. L'abstinent échange des produits actuels contre des produits futurs. Il échange du blé aujourd'hui contre du blé demain. Il est le prêteur. Il s'abstient non pas parce qu'il souhaite toucher un revenu d'intérêt, mais parce qu'il déprécie le présent et apprécie le futur. L'évolution du niveau de l'épargne traduit donc l'évolution du taux d'intérêt originaire (préférence temporelle). Plus les individus déprécient le présent plus ils s'abstiennent de consommer. Le montant des biens épargnés croît alors avec le taux d'intérêt originaire.

Les banques peuvent alors organiser des échanges différés entre les individus selon leurs préférences temporelles. Les emprunteurs, moyennant le paiement d'un taux d'intérêt des prêts, utilisent aujourd'hui les biens destinés à la consommation future. Le niveau du taux d'intérêt des prêts est d'autant plus élevé que le montant de l'épargne est faible. La rareté de l'épargne informe, par conséquent, les entrepreneurs sur les préférences temporelles des agents. Elle permet de supposer que les hommes préfèrent le présent au futur et incite les entrepreneurs à produire des biens pour la consommation d'aujourd'hui.

Toute baisse des taux d'intérêt des prêts provoque alors un ajustement de la structure du capital à la demande future. Elle informe les entrepreneurs que les agents dévalorisent le présent et leur permet d'anticiper un écart de valeur entre les biens présents et les biens futurs favorable au futur. Elle les incite à réorienter leur production vers le futur puisque la valeur des projets de long terme a augmenté relativement à la valeur des projets de court terme (O'Driscoll et Rizzo 1985, p.205). 
L'ensemble de la structure du capital s'ajuste donc pour exploiter les écarts inter-temporels de valeur. Si l'entrepreneur a correctement anticipé les prix et le type de bien demandé, il réalisera des profits qu'il n'aurait pas réalisés s'il n'avait pas réagi à la variation du taux d'intérêt des prêts et s'il ne s'était pas adapté par un allongement du processus de production aux nouvelles préférences temporelles des agents.

\section{Taux d'intérêt des prêts et variation de la valeur de la monnaie}

En économie de marché l'ensemble de ces calculs est exprimé en monnaie. Tous les biens ont des prix monétaires. Tous les prix sont donc influencés par le prix de la monnaie qui informe les entrepreneurs sur son pouvoir d'achat.

Lors d'un échange différé comme l'acte de prêt, la variation du prix de la monnaie est extrêmement importante. Une baisse du prix de la monnaie altère la répartition des revenus entre les prêteurs et les emprunteurs. Elle spolie les détenteurs de créance au profit de leurs débiteurs. Une hausse du prix de la monnaie a l'effet inverse. Elle spolie les débiteurs par les charges indûment accrues de leurs dettes. Les entrepreneurs sont, dans ces conditions, d'autant mieux informés sur les préférences temporelles des individus que le pouvoir d'achat de la monnaie (son prix) est stable dans le temps, car dans le cas contraire, le taux d'intérêt des prêts variera indépendamment du taux d'intérêt originaire. C'est parce que les entrepreneurs ne peuvent pas faire la différence entre une hausse du taux d'intérêt des prêts induite par une variation de la quantité d'épargne (variation du taux d'intérêt originaire) et une hausse induite par une variation du prix de la monnaie qu'ils vont prendre des décisions inadaptées à la demande des consommateurs. Cette baisse artificielle des taux d'intérêt provoque alors une 
distorsion générale de la hiérarchie des prix relatifs qui induit un mécanisme d'auto-renforcement des erreurs et une mauvaise coordination des actions humaines sur le marché. Ce sont donc les banquiers qui engagent l'ensemble des entrepreneurs dans un processus d'ajustement de la structure du capital qui n'a pas lieu d'être.

\section{Variation du prix de la monnaie et cycle}

Les entrepreneurs interprètent la baisse du taux d'intérêt des prêts comme le signe d'une appréciation du futur. Ils ont tendance, pour cette raison, à réorienter la structure du capital vers les projets de long terme au détriment des projets de court terme. Ils se préparent ainsi à répondre à une demande de biens futurs, alors que les consommateurs n'ont pas changé leur préférence temporelle. La création de crédits non gagés sur de l'épargne en monnaie est à l'origine d'une phase d'expansion. Elle est aussi la cause de l'inadaptation de la structure de la production à la demande et de la phase de récession.

La séquence de la théorie autrichienne des cycles est la suivante. La multiplication des projets de production de plus long terme (produire pour le futur) induite par la baisse artificielle des taux d'intérêt des prêts provoque une pénurie de biens de consommation qui favorise un mouvement de hausse des prix des biens de consommation (Hayek 1931, p.118). Cette tension inflationniste force les consommateurs à consommer moins et à épargner plus. La hausse des prix des biens équivaut alors à une épargne forcée (Hayek 1932). Les consommateurs subissent les décisions des entrepreneurs sans bénéficier des gains de productivité (Hayek 1931, p.116). Cette tension inflationniste ne concerne cependant pas tous les biens immédiatement. L'augmentation des prix, avant d'être générale comme dans la théorie monétariste, dépend du lieu d'entrée et 
de sortie de la monnaie dans le système économique (effet Cantillon). Les entrepreneurs qui ont obtenu un prêt dépensent leur argent et créent une offre additionnelle qui accroît d'abord les revenus des personnes qui produisent les biens demandés par les emprunteurs.

Outre l'augmentation du prix des biens de consommation, la préparation de l'offre de biens pour le futur augmente le prix des facteurs de production (effet Ricardo). Les salaires ont alors tendance à augmenter dans ces secteurs et à favoriser une augmentation des coûts de production dans l'ensemble de l'économie. La production des biens de consommation diminue alors au fur et à mesure que les projets de long terme attirent le travail et les autres facteurs de production. L'absence d'augmentation du montant de l'épargne volontaire crée donc un déséquilibre entre les investissements $e x$ ante et l'épargne $e x$ ante qui se résorbe $e x$ post par un phénomène d'épargne forcée.

Le premier effet de l'injection de crédits artificiels est de frustrer les plans de consommation. La coordination est mauvaise puisque les consommateurs sont obligés de diminuer leur niveau de consommation présente pour préparer une consommation future qu'ils ne souhaitent pas. Contrairement à la croissance provoquée par une augmentation de l'épargne, l'investissement des entrepreneurs dans des projets de plus long terme se fait désormais au détriment de la consommation qui doit mobiliser une plus grande quantité de monnaie pour acheter une même quantité de bien.

Tant que du crédit artificiel est injecté, le mouvement vers les projets de long terme et l'épargne forcée se perpétuent. L'augmentation du volume des moyens de paiement s'avère cependant insoutenable sur le long terme pour deux raisons. Les consommateurs ne peuvent pas rester dans cet état de frustration et les banques ne peuvent pas créer de la monnaie indéfiniment. Le caractère involontaire de l'ensemble de ces modifications rend les systèmes économiques vulnérables à un retour à des 
processus de production moins longs. Ce retour explique les crises (Hayek 1931, p.118). L'expansion va alors laisser place à la récession car la structure du capital induite par l'apport de moyens de paiement est inconsistante avec les préférences pour le temps des individus. Il y a mauvaise coordination intertemporelle parce que la baisse artificielle du taux d'intérêt des prêts a favorisé un phénomène de mal-investissement (Mises 1985, p586). Il y a sur-investissement en bien de capital et sousinvestissement en bien de consommation.

La crise survient quand les banques ne peuvent plus assurer le financement des investissements longs qui s'avèrent moins profitables que prévu. Les entrepreneurs constatent que leurs anticipations sont erronées. Le volume de biens futurs n'est pas celui qu'ils avaient estimé. «La classe des entrepreneurs est dans la situation d'un maître d'œuvre dont la tâche est d'ériger un bâtiment à partir d'un stock limité de matériaux. Si l'entrepreneur estime qu'il a plus de matériaux qu'il n'en a en réalité, il dresse un plan qui dépasse ses moyens. Il exagère les dimensions du terrassement et des fondations et plus tard découvre en poursuivant la construction que les matériaux lui manquent pour achever le bâtiment» (Mises 1985, p.586). Ils sont alors obligés de ré-ajuster la structure de la production pour éviter les phénomènes de surproduction. Ils décident de licencier ${ }^{5}$. Si les banques ne peuvent pas les aider financièrement pendant cette période de restructuration, il est probable que la restructuration se termine par un mouvement de faillite qui fragilise l'ensemble du système économique et provoque une profonde récession. Ce scénario s'avère le plus vraisemblable car les banques restent contraintes par un ratio de liquidité. Cette contrainte favorise une hausse des taux d'intérêt qui signale à l'ensemble des agents que les consommateurs préfèrent le présent. Elle sonne le glas de tous les projets qui avaient tablé sur une forte appréciation du futur.

5 Pour l'école autrichienne les causes des cycles sont l'origine principale du chômage massif et permanent d'une partie de la population (Gunning 1985). 
Les cycles économiques sont donc le résultat des décisions des banques qui sont un facteur d'instabilité lorsqu'elles sont à l'origine d'une structure des taux d'intérêt des prêts qui ne reflète pas les taux d'intérêt originaires. Cette explication reste cependant encore relativement imprécise. Elle ne précise pas, tout d'abord, les conditions dans lesquelles les banquiers créent l'excès de monnaie qui provoque une distorsion de la structure des taux d'intérêt. Elle ne dit pas, ensuite, pourquoi les erreurs $\mathrm{d}$ 'anticipation sont immuablement récurrentes et pourquoi toutes les banques augmentent leur taux d'intérêt des prêts au même moment. On pourrait, en effet, penser que progressivement les banquiers et les entrepreneurs qui acceptent les prêts savent qu'en accordant trop de crédit ils fragilisent le système monétaire et augmentent les risques de faillite et d'instabilité. Il faut alors expliquer pourquoi les individus sont conscients de leurs erreurs ou des conséquences de leurs erreurs, mais ne souhaitent pas les corriger (Hülsmann 1998, p.9).

\section{CYCLES ÉCONOMIQUES, CONDITIONS INSTITUTIONNELLES DE L'ACTIVITÉ BANCAIRE ET EXCÈS DE MONNAIE}

Les banquiers créent un excès d'offre de monnaie qui provoque une distorsion de la structure des taux d'intérêt parce qu'ils agissent dans le cadre d'un système monétaire hiérarchisé autour d'une banque centrale (Hayek 1978) ${ }^{6}$. Cette explication générique cache cependant un débat sur la manière dont la monopolisation de l'offre de monnaie par les Etats modifie le comportement des banques et conduit à l'excès d'offre de monnaie qui provoque le cycle.

${ }^{6}$ Voir pour une brève histoire de la nationalisation de la monnaie en France le livre de Salin (1991) et l'article de Nataf (1997). 
Un premier groupe d'économistes autrichiens, réuni sous la bannière de l'école de la libre circulation, soutient que c'est la généralisation de la pratique des réserves fractionnaires qui explique la distorsion de la structure des taux d'intérêt. Cette pratique explique pourquoi l'argent prêté ne correspond pas nécessairement à l'argent déposé en banque à cet effet. Les cycles et l'instabilité de l'économie de marché disparaîtraient si les banques n'avaient pas le droit de créer des moyens de paiement non convertibles en monnaie. L'école de la libre circulation s'accorde, pour cette raison, avec l'école de la circulation pour insérer l'émission de billets dans des limites très strictes ${ }^{7}$, mais estime que seule la dénationalisation de la monnaie permet de réaliser cet objectif.

Un deuxième groupe, plus proche des positions de l'école de la banque ${ }^{8}$, conteste la thèse du coefficient de réserve de $100 \%$ et explique les déséquilibres monétaires par l'absence de concurrence. Cette école dite de la banque libre soutient que les cycles ne sont pas liés à l'émission de moyens de paiement non convertibles par les banques et que de telles pratiques ont plutôt tendance à éliminer les récessions économiques 9 .

${ }^{7}$ L'école de la circulation («currency school ») adopte les thèses bullionistes et soutient que la monnaie ne détermine que le niveau des prix et les cours du change. Elle devait donc être sévèrement contrôlée et n'être émise qu'en contrepartie d'un accroissement du stock d'or national, à la suite d'un excédent de la balance commerciale et d'une hausse du cours du change (de Mourgues 1993, p.172).

${ }^{8}$ L'école de la banque ("Banking school») soutient que l'émission de monnaie devait être laissée libre et qu'elle ne présentait aucun danger si elle correspondait à des créances sûres et à court terme qui permettaient à la banque d'émission de contrôler sa production et de rectifier ses erreurs (de Mourgues 1993, p.172).

${ }^{9}$ Salin (2001) combat la thèse des réserves fractionnaires. Il propose, cependant, de scinder le débat en trois. Il juge que les raisons avancées par l'école de la banque libre (Selgin et White, 1996) sont incorrectes, mais soutient que les systèmes monétaires avec réserves fractionnaires sont stables. 


\section{L'école de la libre circulation: la pratique des réserves fractionnaires est à l'origine des cycles}

Pour l'école de la libre circulation, l'étude des causes et des effets de la pratique des réserves fractionnaires permet de préciser les conditions dans lesquelles les banques sont à l'origine des cycles. Cette pratique explique l'erreur d'anticipation des banques et la baisse artificielle des taux d'intérêt des prêts. Il y a réserve fractionnaire lorsqu'une banque émet de la monnaie non convertible (fiduciary media).

Sous les catégories forgées par Ludwig von Mises ${ }^{10}$ (1966, pp.433), la monnaie non convertible est une forme particulière de monnaie. Mises distingue, en effet, le crédit réel (crédit gagé sur l'épargne) et le crédit artificiel (crédit pur). Les banques émettent des moyens de paiement (billets et chèques) qui ne sont pas de la monnaie proprement dit, mais des substituts monétaires (money substitutes). Ces substituts monétaires sont des créances émises par les banques qui sont à l'origine payables et remboursables à vue et au porteur en un bien économique ayant sa valeur propre (or ou argent). Les moyens de paiement sont convertibles en monnaie. Lorsqu'un moyen de paiement n'est pas totalement convertible, il est utile de distinguer la partie convertible que Mises (1966, pp.434) appelle un certificat monétaire et la partie non convertible que Mises appelle un moyen de paiement fiduciaire (fiduciary media) ${ }^{11}$. Il ne faut pas,

${ }^{10}$ La place de Mises dans ce débat n'est pas encore vraiment élucidée. Salerno (1993) estime que Mises n'est pas partisan de la banque libre. Il soutient que Mises développe une position orthodoxe (pour le 100\%). La controverse entre Le Maux (2000) et Hülsmann (2000a) traite spécifiquement de cette question. Le Maux (2000) juge, en effet, que Mises ne soutient pas la règle des 100\%. Voir aussi la controverse entre Selgin (2000) qui considère que Mises n'a pas vraiment pris parti et qu'il y voit des avantages et des inconvénients et Hülsmann (2000b). Nous ne présentons cependant pas ce débat dans le corps du texte, car nous partageons la position de Selgin (2000, p.94) qui pense que le problème ne doit pas être résolu par un argument d'autorité, " Mises a dit ", mais par un effort d'argumentation original.

11 Voir Salerno J.T. (1994, p.75-77). 
pour cette raison, assimiler mécaniquement billets de banque et monnaie comme le fait l'école de la circulation (currency school) puisqu'il peut dans certain cas exister des moyens de paiement fiduciares (partie non convertible). Il ne faut pas non plus soutenir, comme l'école de la banque (banking school), que les billets ne sont que des créances sur la banque et que rien ne garantit pour cette raison leur convertibilité puisqu'il existe des certificats monétaires (partie convertible).

$C^{\prime}$ est parce que la nationalisation de l'offre de monnaie incite les banques à émettre de la monnaie non convertible qu'elle est à l'origine des cycles économiques et d'importants effets dominos.

\section{a) Réserve fractionnaire et cycles économiques}

Pour que le taux d'intérêt des prêts reflète les préférences temporelles des prêteurs, il faut que les banques ne confondent pas l'argent déposé et l'argent épargné.

La banque rend, en effet, deux types de service. Elle vend son coffre et met en relation les prêteurs et les emprunteurs.

Le client du banquier achète, tout d'abord, aux banquiers les services rendus par son coffre fort. Il met une partie de ses ressources à la banque pour des raisons de sécurité (Hüslmann 2000b). La banque n'est pas censée utiliser ses dépôts en monnaie. Le client de la banque obtient un certificat de dépôt qu'il peut utiliser pour acheter des biens et services (monnaie de transaction) et faciliter ainsi ses transactions. Il peut aussi exiger la remise immédiate de sa monnaie afin de profiter d'un prix intéressant sur le marché. La banque est donc contractuellement tenue de ne pas utiliser la monnaie ainsi déposée. Elle est dans l'incapacité juridique de prêter ce qu'elle ne possède pas.

Le client $\mathrm{du}$ banquier achète, ensuite, un service $\mathrm{d}$ 'intermédiation. Le banquier met le prêteur en relation avec 
l'emprunteur. Il informe l'emprunteur des choix inter-temporels du prêteur. Le prêteur vend alors un service à l'emprunteur par l'intermédiaire de la banque qui met en relation les deux parties au contrat. Avant tout contrat, le prêteur informe le banquier $\mathrm{du}$ montant de monnaie qu'il souhaite garder pour sa consommation courante et le montant de monnaie qu'il souhaite affecter à la consommation future. Le prêteur sait, en effet, qu'une fois prêté, son argent ne lui appartiendra plus et qu'il faudra qu'il attende la fin du contrat ou le remboursement du prêt pour pouvoir à nouveau utiliser cette monnaie comme il l'entend. La monnaie prêtée devient la propriété de l'emprunteur. Le banquier est, dans ces conditions, informé d'une partie des choix inter-temporels du prêteur. L'action de prêter révèle au banquier une préférence pour le futur. Une augmentation de l'offre de monnaie affectée au prêt est donc interprétée comme une valorisation du futur. L'information véhiculée par le prix des prêts est une bonne approximation de la préférence temporelle des individus.

Lorsque le banquier pratique le système des réserves fractionnaires, il prête de l'argent qui n'a pas été épargné, mais seulement déposé. Prenons un exemple. Un individu (A) dépose 100 Euros dans les coffres de la banque $X$ pour s'assurer contre le vol. La banque $X$ utilise 50 de ces 100 Euros pour accorder un crédit à un individu (B). La banque prête ainsi à l'individu B sans que l'individu $\mathrm{A}$ ait renoncé à sa monnaie. Elle crée donc de la monnaie. Elle fait croire aux individus (A) et (B) qu'ils peuvent immédiatement disposer d'un montant total de 150 Euros alors qu'il n'y a que 100 Euros déposés à la banque. Le banquier, dans ce cas, développe une activité risquée, car il distribue des crédits instables, c'est-à-dire des crédits qui ne sont pas garantis par de la monnaie. Il octroie des crédits qui ne sont gagés sur aucune décision d'épargne en monnaie. Le crédit pur s'engendre de lui-même. L'argent ne naît pas d'un argent qui lui préexisterait. Il est crée ex nihilo. Les prêts font les dépôts 
qui font les prêts qui font les dépôts, etc. C'est pour cette raison que le banquier est à l'origine des cycles. Il prête plus de monnaie qu'il n'a le droit (épargne monétaire) afin d'accroître son niveau d'activité. Il baisse ainsi le taux d'intérêt des prêts, injecte de la monnaie dans l'économie et biaise l'information véhiculée par le système des prix en éloignant les entrepreneurs des choix inter-temporels des acteurs. Les emprunteurs profitent, par conséquent, de ce Knut Wicksell appelle l'épargne forcée parce que les banques peuvent offrir plus de crédit qu'elles ne possèdent d'épargne monétaire.

La monnaie-crédit non gagée sur de l'épargne monétaire tend alors à biaiser l'information véhiculée par les taux d'intérêt des prêts et à favoriser un processus de mauvaise coordination qui ne trouve sa solution que dans une profonde phase de récession.

\section{b) La pratique des réserves fractionnaires expose l'économie de marché à des effets dominos}

La pratique des réserves fractionnaires accroît aussi l'instabilité des économies de marché parce qu'elle l'expose à des effets dominos. Une banque fait faillite parce qu'elle ne peut pas répondre à la demande de monnaie et entraîne avec elle d'autres banques, ce qui déstabilise complètement le système monétaire et financier et biaise l'ensemble de la structure du capital qui devient totalement inadéquate. La théorie autrichienne des cycles précise les étapes de cet effet. L'école de la libre circulation soutient donc que l'origine des crises s'explique par la pratique frauduleuse des réserves fractionnaires et que cette dernière explique à son tour la création d'une banque centrale (de Soto 1995, p.32). Car lorsque les banques ont compris qu'il était très lucratif de violer les principes des droits de propriété qui règle le système du $100 \%$, elles ont fait pression sur le gouvernement pour qu'il joue le rôle du prêteur en dernier ressort ou/et qu'il délègue cette fonction à une banque centrale dont la mission est 
de fournir les liquidités lorsqu'elles ont trop émis de moyens de paiement fiduciaires (fiduciary media).

\section{c) La pratique des réserves fractionnaires est frauduleuse}

Cette pratique ne serait, cependant, pas possible dans les systèmes monétaires de libre circulation. Dans un système monétaire non hiérarchisé, les banques qui violent «le principe $d u$ droit selon lequel dans le contrat de dépôt d'argent fongible, l'obligation traditionnelle de garde qui est un élément essentiel dans tout dépôt non fongible, se matérialise dans l'exigence de maintenir, à tout moment, une réserve de 100 pour cent de la quantité d'argent fongible reçue en dépôt, (...)» (Huerta de Soto 1994, p.383) seront sanctionnées par la justice. La règle du $100 \%$ de réserve s'impose par conséquent spontanément comme une règle abstraite parce qu'elle est la simple application du droit de propriété privée (Huerta de Soto 1994, p.388, note 18) ${ }^{12}$. La pratique des réserves fractionnaires est frauduleuse (Huerta de Soto 1994, p.386) ${ }^{13}$ parce que le banquier agit sans le consentement de son client qui a déposé sa monnaie, non pas pour un prêt, mais uniquement pour faciliter sa disponibilité monétaire (Huerta de Soto 1994, Hoppe et al. $1998^{14}$, Huerta de Soto 1998b, p.41).

La pratique des réserves fractionnaires enrichit donc les banques et une partie de la population aux dépens de $l^{\prime}$ autre ${ }^{15}$.

${ }^{12}$ Huerta de Soto (1994, p.384 note 9) rappelle qu'un jugement du Tribunal de Paris du 12 juin 1927 condamna un banquier pour délit d'appropriation frauduleuse pour avoir utilisé, selon la pratique bancaire commune, les fonds qu'il avait reçus en dépôt de son client. Une autre sentence du même tribunal du 4 janvier 1934 se prononce dans le même sens.

13 Huerta de Soto $(1997$, p.4) remarque que cet argument est déjà utilisé par Navarro en 1556 dans son livre, Commentaire sur l'échange.

14 Hoppe et al. (1998) soutiennent que les propriétaires de la monnaie crédit pur (emprunteur) ne sont pas les victimes de la fraude, mais des conspirateurs. Ils aident la banque à détourner la loi.

15 L'institution des banques centrales est le résultat d'une action coercitive des gouvernements. Elle est le résultat de la complicité du gouvernement des banques pour institutionnaliser le système des réserves fractionnaires (Huerta de Soto 1995, p.28). 
1) Elle explique le caractère cyclique des économies de marché avec banque centrale, 2) viole les droits de propriété, et 3 ) favorise l'instabilité et la méfiance.

\section{L'école de la banque libre: les cycles s'expliquent par un déséquilibre monétaire}

L'école de la banque libre (free banking school) ne renie pas les distinctions proposées par Mises, mais estime que la pratique des réserves fractionnaires n'est pas à l'origine de l'instabilité du prix de la monnaie. Elle soutient ensuite que 1) la concurrence entre les monnaies assure la stabilité économique, que 2) la pratique des réserves fractionnaires respecte la liberté contractuelle et que 3) l'existence d'un marché inter-bancaire garantit la stabilité des systèmes de banque libre. Elle mobilise aussi l'histoire et estime que les banques ont toujours cherché à contourner le coefficient de réserve de $100 \%$, que le contrôle de la base monétaire reste précaire (Yeager 2001, p.257) et que les systèmes de banque libre sont stables alors qu'ils ne pratiquent pas le coefficient de réserve de $100 \%$.

\section{a) Cycles économiques et déséquilibres monétaires}

L'école de la banque libre n'explique pas, tout d'abord, les cycles par l'absence momentanée de liquidité des banques, mais par les déséquilibres monétaires. Elle est, sur ce point, en accord avec l'école de la banque. Elle s'en écarte, cependant, lorsqu'elle estime que les déséquilibres monétaires sont le résultat du monopole de la création monétaire accordé aux banques centrales. Elle propose une théorie des déséquilibres monétaires où l'excès de monnaie est mesuré par rapport à la demande de monnaie et non par rapport à la quantité d'épargne monétaire (Selgin et White 1996). Son raisonnement est le suivant. 
Elle débute par le problème classique de la compatibilité de l'équilibre monétaire (offre et demande de fonds prêtables) et de l'égalité épargne - investissement (Selgin 1991, p.91). Elle soutient, ensuite, que «la demande globale d'avoirs en monnaie-banque reflète le consentement du public à fournir des fonds prêtables par l'intermédiaire des banques sur lesquelles il détient ses créances». Les banques sont, dans ces conditions, de simples intermédiaires pour les fonds prêtables (p.92). Elles créent une relation directe entre les conditions d'équilibre sur le marché des avoirs en monnaie-banque et les conditions d'équilibre sur le marché des fonds prêtables. Toute augmentation de la demande de monnaie justifie alors une augmentation des prêts et des investissements bancaires. La phase de récession qui engage le cycle s'explique, dans ces conditions, par un déséquilibre monétaire. Si une hausse de l'offre de monnaie crée un excès de monnaie, les gens dépenseront cet excès (Selgin 2000, p.97). «Si les salariés souhaitent plus de monnaie mais que les banques ne répondent pas à cette demande ils baisseront leurs achats de biens de consommation» (Selgin 1991, p.92). Les entrepreneurs interprèteront cette baisse de la consommation comme une baisse durable de la rentabilité de leurs activités. Ils réduiront, alors, leur capacité de production et provoqueront le développement d'une récession. La phase de récession est donc le résultat d'un mauvais ajustement de l'offre à la demande de monnaie. Une crise ne peut se produire que si les banques ne répondent pas de façon appropriée à une hausse de la demande de monnaie.

L'originalité de la théorie de la banque libre ne se trouve pas, cependant, dans cette explication monétaire des phases de récession. Elle est plutôt dans l'approfondissement de la théorie hayékienne de la dénationalisation de la monnaie (Hayek 1978). Hayek a, en effet, avancé l'idée que la stabilité du niveau général des prix sera réalisée dans de meilleures conditions dans un régime de concurrence que dans un régime de monopole. C'est sur cette base que l'école de la banque libre explique l'ampleur des déséquilibres monétaires constatés dans les économies de 
marché. Après avoir rappelé l'horizon de long terme qui entoure la gestion de la masse monétaire (Selgin 1991, Chapitre 7), l'absence de coûts physiques de production de la monnaie et ses conséquences sur les problèmes d'information rencontrés par les banques pour ajuster l'offre et la demande de monnaie, les théoriciens de la banque libre soutiennent que la concurrence entre les banques est un processus de découverte de l'offre de monnaie plus performant que le monopole. Toute la théorie des processus de marché est mobilisée pour atteindre cette conclusion qui repose simplement sur l'idée qu'un système centralisé est moins apte à mobiliser l'information tacite qui existe à l'état synthétique dans le système des prix.

\section{b) La pratique des réserves fractionnaires respecte la liberté contractuelle}

L'école de la banque libre soutient, ensuite, que la pratique des réserves fractionnaires n'est pas un acte illégal car rien n'indique que les clients des banques tiennent à ce que les banques pratiquent un coefficient de réserve de $100 \%$. Les réserves fractionnaires ne sont frauduleuses que parce que la demande des clients se porte sur les banques qui pratiquent le $100 \%$. Cette hypothèse n'est, cependant, étayée par rien et ne peut surtout pas être généralisée à l'ensemble des agents.

Elles ne sont pas non plus frauduleuses parce qu'émettre un billet de banque oblige le banquier à rembourser le porteur (Le Maux 2000, p.173). Le banquier ne s'engage pas à redonner le même dépôt or, mais une même quantité. Il n'y a donc rien de frauduleux dans cette pratique.

S'il n'est pas frauduleux de pratiquer le système des réserves fractionnaires, alors interdire ce type de contrat est liberticide (White 1989, p.156, Salin 1998). L'absence de banque centrale ne se traduira plus par la généralisation de la règle du $100 \%$ mais par le cloisonnement du système bancaire; d'un côté les 
banques qui affichent leur coefficient de réserve de $100 \%$ et de l'autre les banques qui pratiquent des réserves fractionnaires (Selgin 2000, p.96).

c) Les systèmes de banque libre ne sont pas menacés par des effets dominos

L'école de la banque libre admet enfin le risque que prend la banque à prêter plus de monnaie qu'elle n'en a en banque (Selgin 2000, p.18) mais estime qu'il faut se poser la question de savoir si «le jeu en vaut la chandelle». Les systèmes de banque libre avec réserves fractionnaires prennent le risque que tous les clients d'une banque demandent le remboursement de leur créance (fiduciary medias) au même moment. Cette perte de confiance généralisée serait à l'évidence à l'origine d'une crise et d'une forte instabilité du système économique.

L'école de la banque libre estime, cependant, que seule une défaillance du marché inter-bancaire de prêts et emprunts de très courte durée (le jour) peut provoquer une telle crise d'illiquidité. Cet échec n'est pas vraisemblable (Salin 1991, Chapitre IV, Selgin 1991), car dés qu'une banque apparaît comme un risque, elle ne trouve plus de prêteur. A cet argument s'en ajoute deux autres.1) Si une banque n'a qu'un client, le risque qu'il vienne un jour demander la conversion de la totalité de ses certificats en termes d'or est grand. Le banquier est, dans ces conditions, obligé de respecter le $100 \%$ or. Mais si le nombre des clients est important, la loi des grands nombres joue et assure que tous les déposants ne viendront pas en même temps chercher leur or (Salin 1991, p.67). 2) Les banques ont ensuite intérêt à constituer un système bancaire afin de garantir la convertibilité de leur monnaie et d'accroître ainsi l'utilité de la monnaie pour leur détenteur (Salin 1991, p.85). L'une des conséquences de ce système est la mise en œuvre d'un marché où les banques s'échangent leur monnaie à un taux convenu contractuellement. 
Une banque qui émettrait trop de billets par rapport au nombre de ses déposants et qui accroîtrait ses risques d'illiquidité serait sanctionnée par les tribunaux si elle changeait son taux de conversion or. Les créanciers pourraient cependant l'accepter pour éviter la faillite (Salin 1991, p.89). Si la faillite est inévitable, ils pourraient se répartir les coûts et les répercuter en fonction de leur contrainte sur le prix de leur service. Les banquiers, pour prévenir ces situations, peuvent aussi instituer des règles de bonne conduite. Ces règles porteront sur les coefficients de réserve. Tout banquier qui ne respecte pas un certain niveau de réserve sera exclu du système ou financièrement sanctionné. Le système bancaire, qui n'est rien d'autre qu'un cartel spontané, développe des stratégies pour endiguer les effets de dominos et conserver leur avantage concurrentiel vis-à-vis d'autres cartels de banque contrôlant d'autres zones territoriales (Salin 1991, 1998).

\section{Ecole de la Banque libre versus Ecole de la libre circulation}

Les critiques de l'école de la banque libre ont logiquement donné lieu à des réponses de l'école de la libre circulation qui a étayé ses arguments et tenté de persuader de la légitimité de sa position.

\section{a) Réserves fractionnaires versus déséquilibres monétaires}

L'école de la libre circulation a tout d'abord estimé que l'école de la banque libre confondait demande de monnaie et épargne en monnaie (Hoppe 1994, pp.71-73, Huerta de Soto 1998b, p.3537) et oubliait que l'offre et la demande de monnaie s'ajustaient aussi par le pouvoir d'achat de monnaie (Salerno 1983, p.246). Il n'est pas juste, en effet, de soutenir qu'une demande de 
monnaie supplémentaire sans offre nouvelle de monnaie conduit nécessairement à un déséquilibre car l'offre de monnaie ne s'ajuste pas uniquement par les quantités. Elle s'ajuste aussi par les prix. La stabilité de l'offre de monnaie provoque une hausse du pouvoir d'achat de la monnaie et de la rentabilité de l'extraction de l'or (Salerno 1983).

Il n'est pas correct, ensuite, de soutenir que l'augmentation de la demande de monnaie équivaut à une hausse de l'épargne dès que la banque a ouvert une ligne de crédit à son client. Cette position conduit les économistes de la banque libre à oublier l'importance des ajustements inter-temporels sur le marché. L'augmentation de la demande de monnaie n'équivaut pas à une augmentation de l'épargne parce que l'épargne traduit les préférences temporelles des hommes alors que la demande de monnaie informe sur son utilité. Cette différence rappelle qu'il ne faut pas traiter tous les dépôts en monnaie comme de l'épargne car une partie de la demande de monnaie répond à une demande de liquidités. Une augmentation de la demande de monnaie ne signifie pas, dans ces conditions, une hausse de l'épargne, c'est-à-dire un changement des préférences temporelles des agents. Elle ne doit pas non plus être interprétée comme une évolution des préférences temporelles. Il peut juste s'agir d'une augmentation de la demande de liquidité. Il est logique, à la suite de ce raisonnement, de soutenir que seul le respect du $100 \%$ permet au taux d'intérêt des banques de refléter les préférences temporelles. Dès qu'apparaissent les moyens de paiement fiduciaires les taux d'intérêt ne reflètent plus les préférences temporelles et la structure du capital devient inadaptée. 


\section{b) Liberté contractuelle versus Droits de propriété}

Le débat sur l'illégalité des réserves fractionnaires dans un système non hiérarchisé a aussi fait l'objet de nombreux échanges. Hoppe (1994, p.70) estime que l'école de la banque libre se méprend sur la notion de liberté contractuelle. La liberté contractuelle ne signifie pas que deux individus s'entendent pour en voler un troisième ${ }^{16}$.

Cet argument est juste mais il n'entre pas dans la logique de l'école de la banque libre qui estime qu'offrir et demander des moyens de paiement fiduciaires n'est pas un crime. Il s'agit seulement d'un contrat. Si un tiers (déposant) se trouve lésé, rien ne l'empêche de faire un recours en justice. Cette solution est d'ailleurs inscrite dans les institutions du marché. L'opposition entre les deux arguments est donc factice car rien $\mathrm{n}^{\prime}$ empêche les banques de pratiquer les réserves fractionnaires ${ }^{17}$. On peut affirmer avec certitude que le système de banque libre sélectionnera les banques en fonction de leur stratégie de différenciation (Hülsmann 2000c) ${ }^{18}$, mais rien ne permet de dire

${ }^{16}$ Ce n'est pas, cependant, parce que les banquiers ont toujours souhaité utiliser l'argent de leurs déposants à leur profit (Huerta de Soto 1995, p.31) que la règle du $100 \%$ n'est pas praticable. Le vol est une activité lucrative que les autorités publiques réussissent malgré tout à juguler.

${ }^{17} \mathrm{Il}$ n'est pas facile cependant d'expliquer les raisons qui pousseraient un client à accepter ce type de pratique. Personne $a$ priori n'acceptera de prêter son argent sans le paiement d'une prime de risque. Il y a bien liberté des contrats, mais il n'y a aucune raison que le déposant accepte un tel contrat qui est désavantageux pour lui. S'il veut prêter son argent, il fait payer le service. Le contrat porte alors sur le taux d'intérêt du prêt et non sur la possibilité de prêter un argent qu'a priori il souhaite immédiatement disponible. La banque qui pratique les réserves fractionnaires le fera donc bien sans le consentement de son client (sauf exception).

18 L'école de la libre circulation tente, enfin, de développer une voie de conciliation en proposant une théorie de la différenciation. Le coefficient de réserve deviendrait un facteur de discrimination important pour les clients qui, selon leur besoin et leur degré d'aversion au risque s'adresseront à des banques pratiquant les réserves fractionnaires ou à des banques respectant la règle du 100\% (Hüslmann 2000c). Cette position est déjà tenue par Salerno (1983) qui propose de distinguer les banques des institutions financières afin de sortir du débat sur les réserves fractionnaires de l'impasse. 
avec certitude si ce sont les banques qui pratiquent un coefficient de réserve de $100 \%$ qui passeront le test du marché. Seule la mise en place de systèmes monétaires non hiérarchisés sur longue période permettrait de l'affirmer.

c) Effets de dominos versus stabilité des systèmes de banque libre

Le troisième argument débattu porte sur la stabilité des systèmes de banque libre avec réserves fractionnaires. L'histoire est mobilisée par les deux écoles. L'école de la libre circulation prend argument de la disparition des systèmes de banque libre pour valider sa position ${ }^{19}$. Si toutes les expériences historiques de banque libre avec réserves fractionnaires ont échoué (Huerta de Soto 1998, pp.37-39) et conduit à l'institutionnalisation d'une banque centrale, c'est qu'elles n'étaient pas viables. Les systèmes de banque libre avec réserves fractionnaires n'auraient donc pas passé le test du marché (Hülsmann 2000c).

L'école de la banque libre rejette cette position en montrant que les systèmes de banque libre étaient performants et stables (Selgin 1988, Chapitre 1 et p.243). Elle établit un certain nombre de faits (Dowd 1992, 1994). Le système de banque libre favorise une grande stabilité du pouvoir d'achat de la monnaie et fonctionne plutôt sur une base matérielle qui est l'or. Aucun système de banque libre n'a jamais abandonné la convertibilité or et aucune évidence historique ne permet d'affirmer que la concurrence entre les banques favorise l'explosion monétaire et l'accélération de l'inflation. Ces systèmes étaient très stables et l'offre supplémentaire de monnaie induite par les découvertes de mines d'or était rapidement intégrée dans le système (White 1984). Leur stabilité était caractérisée par de faibles variations des taux d'intérêt dans les systèmes écossais et canadiens, par

19 Voir par exemple Rothbard (1988) pour l'école de la libre circulation et Horwitz (1993) pour l'école de la banque libre. 
une meilleure capacité à réagir que les systèmes américains ou anglais face aux chocs climatiques (Dowd 1994, p.411) et par un financement sur fonds permanents beaucoup plus élevé dans les systèmes libres que dans les systèmes réglementés ou hiérarchisés ${ }^{20}$. On peut en conclure que vraisemblablement dans un système de banque libre les banques mettront en place un système de compensation qui n'empêchera pas une banque de faire faillite si elle a pris des risques inconsidérés, mais qui rendra presque nuls les effets dominos.

Ce n'est donc pas parce que les systèmes de banque libre n'étaient pas viables qu'ils ont disparu, mais parce que les gouvernements politiques souhaitaient pratiquer le seigneuriage, financer ainsi leurs dépenses militaires et leurs stratégies de prédation et répondre à la pression des banquiers.

Les arguments théoriques sont aussi renouvelés. L'école de la libre circulation considère que la constitution d'un cartel de banques permet de soutenir que les risques d'un effet de dominos sont limités mais pas écartés (Hülsmann 1998b). Il est correct aussi de penser que la contrepartie d'un système de parfaite convertibilité des monnaies-banques entre elles est de généraliser les comportements de passager clandestin car dans un tel système, le pouvoir d'achat d'une monnaie-banque dépend du pouvoir d'achat de toutes les autres. Chaque banque est, dans ces conditions, obligée de défendre le cours de la monnaie-banque de sa concurrente pour éviter une baisse du pouvoir d'achat de sa propre monnaie. La mutualisation des risques induite par le cartel a donc un effet pervers puisqu'elle favorise une baisse progressive des coefficients de réserve qui, à terme, ne peut que fragiliser le système monétaire.

L'école autrichienne explique donc l'excès de monnaie par l'institutionnalisation d'un système monétaire hiérarchisé mais se divise sur les conditions de stabilité d'un système de banque

${ }^{20}$ Tous ces résultats sont confirmés par Gentier (2000). 
libre. L'école de la libre circulation soutient que l'absence de banque centrale aurait pour effet d'empêcher la pratique des réserves fractionnaires, de biaiser les taux d'intérêt et de distordre la structure du capital. L'école de la banque libre argue que les systèmes de banque libre pratiqueraient des coefficients de réserve inférieurs à $100 \%$ et que c'est la concurrence qui protégerait la stabilité du système.

\section{UNE EXPLICATION DE LA RÉCURRENCE DES ERREURS COLLECTIVES D'ANTICIPATION}

La théorie autrichienne des cycles ne doit pas cependant se contenter d'expliquer les erreurs d'anticipation qui distordent la structure du capital. Elle doit aussi rendre compte de la récurrence des erreurs et de leur dimension collective, car un cycle se répète par définition et n'est pas composé que d'une phase de récession.

Pour expliquer la récurrence d'erreurs collectives d'anticipation les économistes autrichiens mobilisent leur théorie des anticipations cognitives et leur théorie des droits de propriété. Ils soutiennent que les banquiers n'apprennent rien de leurs erreurs (récurrence) parce qu'ils n'y ont pas intérêt et qu'ils font tous la même erreur parce que l'institutionnalisation d'une banque centrale concentre les anticipations autour de ses décisions. Les pertes de confiance collectives sont, dans ces conditions, amplifiées par la centralisation du système monétaire. 


\section{La socialisation des risques explique la récurrence des erreurs d'anticipation}

La nationalisation de la monnaie favorise des pratiques de prêts non gagés sur de l'épargne en monnaie parce qu'elle tend à socialiser les risques d'une telle pratique. Les banquiers développent largement ce type d'activité parce que le système socialise les pertes sur l'ensemble du système monétaire mais maintient les gains privatifs. Elle limite les effets de l'absence de liquidité sur la faillite des banques et met les banques en face du même choix que les éleveurs en présence d'une pâture commune de libre accès (Huerta de Soto 1995, p.33).

Face à une augmentation de la demande de crédit sans variation de la quantité de monnaie déposée dans les banques (base monétaire), les banques ont deux attitudes possibles, soient elles augmentent le taux d'intérêt des prêts, soient elles baissent leur coefficient de réserve. Si chaque banque est responsable et supporte le coût de l'expansion du crédit, elles ont plutôt tendance à augmenter le taux d'intérêt des prêts. Si les banques peuvent externaliser une partie de leur coût sur les autres banques sans perdre de bénéfices, elles choisiront de réduire leur coefficient de réserve. Chaque banque sait pourtant que réduire le coefficient de réserve accroît les risques de faillite et d'instabilité. Chaque banque a aussi conscience, que si elle ne prête pas pour maintenir le système stable, les autres banques le feront. Il s'agit donc d'un problème classique de dilemme du prisonnier (Carilli et Dempster 2001, p.322).

Lorsque le système institutionnel n'individualise pas correctement les gains et les pertes jointes à une action, il provoque la généralisation des comportements de passager clandestin. En l'absence de banque centrale (prêteur en dernier ressort), chaque banque doit supporter tous les risques de liquidité associés à une diminution du coefficient de réserve. Avec le monopole de la banque centrale, la banque qui prête 
plus qu'elle ne possède en réserve ne supporte pas tous les coûts de son action parce que le prêt supplémentaire n'est qu'une petite partie de l'offre de monnaie du monopole. L'existence d'un prêteur en dernier ressort et d'un monopole de la création de monnaie expliquent donc pourquoi les banques ont intérêt à créer un excès de moyens de paiement non convertibles.

On possède ainsi une explication des récurrences des erreurs collectives. C'est parce que le système institutionnel n'individualise pas correctement les pertes et les profits qu'il suscite la récurrence des erreurs. Les entrepreneurs anticipent toujours de la même manière les effets des politiques monétaires des gouvernements, mais n'ont pas intérêt à en tenir compte, car ils savent que d'autres en profiteront. Autant qu'ils tirent des bénéfices du système avant qu'il n'entre en récession. La phase d'expansion reste bénéfique pour ceux qui ont prêté et emprunté. Les vrais perdants sont ceux qui n'ont pas profité des gains de l'expansion et qui supportent la totalité des pertes pendant la récession. L'existence d'une banque centrale favorise donc l'émergence d'opportunités de profit qui n'existent qu'à l'état latent ou marginal dans un système monétaire qui respecte les droits de propriété absolus des intervenants sur le marché.

\section{L'uniformité des anticipations s'explique par l'existence d'une banque centrale}

Reste pour conclure à expliquer pourquoi les banquiers agissent tous de la même façon.

Dans un système de banque libre, la coordination intertemporelle est le résultat du travail d'anticipation des entrepreneurs. Ces derniers ne sont pas omniscients mais sont aidés dans leur tâche par l'institution des profits monétaires qui permet de savoir relativement rapidement qui a bien anticipé 
la demande car le taux d'intérêt des prêts informe sur la variation du volume de l'épargne mais ne dit rien sur le type de demande qui se développera dans le futur. L'entrepreneur s'en remet alors à son alertness pour définir le type de biens sur lesquels se portera la demande des consommateurs.

Toute variation des taux d'intérêt des prêts (échange différé) informe approximativement les entrepreneurs sur les choix inter-temporels des agents et met l'économie en mouvement. Une modification des préférences temporelles oblige en effet les entrepreneurs à s'adapter et à modifier la structure du capital. Elle provoque des licenciements, des embauches, des pertes et des profits. Elle redistribue les parts de marché et remet en cause les situations acquises par les entrepreneurs parce qu'elle sanctionne les entrepreneurs qui ont échoué dans leur travail de coordination. Une erreur d'anticipation peut par exemple provoquer une sur-évaluation de la valeur des biens futurs et une sous-évaluation de la valeur des biens présents. Les investissements réalisés ne réussissent pas alors à dégager les bénéfices suffisants pour payer l'intérêt des emprunts et les salaires et réaliser des profits.

L'erreur isolée est ainsi à la source de pertes pour l'entrepreneur. Elle n'est cependant qu'une simple source de mouvement lorsqu'elle reste isolée. Le taux d'intérêt des prêts et l'activité des banques n'opèrent, dans ces conditions, pas parfaitement parce qu'ils n'arrivent pas à informer parfaitement les acteurs sur leurs choix inter-temporels. Ils sont cependant des points $\mathrm{d}$ 'orientation indispensables pour l'entrepreneur qui souhaite répondre à la demande future.

L'action de l'investisseur ne correspondra pas toujours à la demande future, mais la diversité des anticipations lui permettra de réajuster son offre en imitant les décisions des entrepreneurs qui auront anticipé la demande des consommateurs avec le plus d'acuité. Cette correction des erreurs d'anticipation isolées se fera par des restructurations industrielles (faillites et rachats, fusions, 
etc.) ou par l'existence d'une structure de production suffisamment flexible pour se réorienter en cas d'erreur d'appréciation de l'entrepreneur sur la nature de la demande future.

La théorie autrichienne des cycles nous met cependant en présence d'erreurs collectives d'anticipation. L'erreur collective est un facteur d'instabilité, car elle suppose que tout le monde se trompe. Il n'existe pas, dans ces conditions, de repères capables de faciliter la réorientation des ressources vers la production des biens qui satisfont la demande. La condition formelle d'une erreur collective est donc la convergence des anticipations (Fehl 1986, p.82).

Développer une théorie des cycles c'est aussi expliquer pourquoi à certaine période de l'Histoire il existe un processus d'auto-renforcement des erreurs d'appréciation sur les besoins futurs des consommateurs.

La théorie des anticipations et l'hypothèse du «big player» sont de bonnes bases pour expliquer l'uniformité des anticipations dans un système monétaire hiérarchisé. L'uniformité des anticipations s'explique par la centralisation du processus de découverte de profit autour des décisions de la banque centrale.

\section{a) La théorie des anticipations}

La théorie des anticipations utilisée ne reprend pas l'hypothèse des anticipations rationnelles de Lucas, elle préfère reprendre et étendre la théorie hayékienne ${ }^{21}$.

Dans la théorie hayékienne, les anticipations sont le résultat d'un travail d'interprétation de la réalité (comme elle est et comme elle pourrait être) encadré et orienté par les règles de l'esprit. Les règles de l'esprit ne sont cependant pas indépendantes de l'environnement dans lequel elles se sont formés. Les anticipations individuelles et les comportements sont dépendants des institutions qui président

\footnotetext{
${ }^{21}$ Cette théorie est construite sur la philosophie de l'esprit exposée par Hayek dans son ouvrage de 1952 Sensory Order.
} 
à l'ordre social (Butos et Koppl 1993, p.317). Toute modification des institutions modifie le contexte dans lequel se forme l'anticipation. Cela signifie que les règles du marché sont intériorisées par les individus qui interprètent la réalité à leur lumière. Ils savent que s'ils ne respectent pas les règles du marché, ils seront sanctionnés. La concurrence sur le marché impose aux participants à l'échange une discipline qui punit les comportements déviants par des pertes.

Cette unicité de la règle ne se traduit cependant pas par une uniformité des comportements, car ce n'est pas parce que tout le monde agit dans le même environnement qu'il aura les mêmes projets. La diversité des plans crée une diversité des anticipations dans un contexte institutionnel identique pour tout le monde. Elle ne se traduit pas non plus par l'uniformité des comportements parce qu'il ne s'agit pas d'un commandement. La règle ne dit pas ce qu'il faut faire dans le détail, mais ce qui est interdit. C'est un principe négatif.

\section{b) L'hypothèse $d u$ «big player»}

Toute modification de l'ordre institutionnel modifie alors les anticipations des acteurs. L'institutionnalisation d'un système monétaire hiérarchisé autour d'une banque centrale est un profond changement du contexte institutionnel des actions humaines sur le marché. Elle introduit un joueur qui a le pouvoir de ne pas se plier à la discipline du marché, autrement dit, un «big player» (Koppl et Yeager 1994). Le «big player» est quelqu'un qui influence les résultats du processus de marché en exerçant un jugement discrétionnaire qui lui permet d'échapper à la logique des pertes et des profits ${ }^{22}$. Le ministre des finances et le gouverneur de la banque centrale sont des «big players».

22 "A Big player in anyone who influences the results of the market process by exercising his discretionary judgement while remaining himself wholly or largely immune from the discipline of profit and loss» (Koppl and Yeager 1992, p.5). 
Quand les marchés sont soumis à l'action discrétionnaire des «big players», ils ne coordonnent plus aussi bien les actions humaines, car l'action des «big players» est moins prédictible que l'action des autres joueurs sur le marché qui obéissent strictement à la loi des pertes et des profits. Tous les entrepreneurs dépendent désormais de l'idiosyncrasie du gouverneur de la banque centrale. Ils doivent, pour cette raison, anticiper ses actions et tenter de prévoir l'imprévisible par rapport aux règles normales du marché. Ils cherchent à interpréter les déclarations, les écrits et l'ensemble des discours des gouverneurs successifs de la banque centrale. Ils redoutent, pour cette raison, tout changement à la tête des banques centrales car ils sont obligés de réapprendre à gérer les personnalités des hommes qui gèrent ces organisations. L'introduction d'un «big player» est aussi un facteur de mauvaise coordination des actions humaines sur le marché parce qu'elle affaiblit le filtre des pertes et des profits. Elle compromet la discipline du marché et la stabilité des anticipations.

\section{c) Action du «big player» et uniformité des anticipations}

L'introduction d'un ««big player» est de surcroît à l'origine d'une tendance à l'uniformisation des anticipations des acteurs car elle centralise le processus d'anticipation autour d'un point focal (la décision de la banque centrale) et transforme le système de règles en un système de commandement.

— Le «big player» centralise le processus d'anticipation. Tous les acteurs sont attentifs à l'action de la banque centrale parce qu'elle a le pouvoir de modifier toute la hiérarchie des taux d'intérêt des prêts. Dans un système hiérarchisé, la nouvelle monnaie est créée lorsque la banque centrale accorde un prêt. Ces prêts sont déposés dans les banques et sont de la responsabilité de la banque centrale. Les banques incitent les emprunteurs (entrepreneurs) à rechercher 
ces prêts par une baisse des taux d'intérêt monétaires. Si elles obtiennent facilement des prêts de la banque centrale, elles peuvent baisser leur taux d'intérêt. Si les prêts de la banque centrale sont coûteux, en revanche, elles augmentent leur taux d'intérêt et limitent le processus de création monétaire. Cela signifie que c'est la politique monétaire menée par la banque centrale qui oriente l'activité des banques. Il ne s'agit plus d'anticiper une demande diffuse constituée d'une infinité d'individus, mais d'anticiper l'action de l'autorité monétaire afin d'en exploiter les effets. Le prix des prêts n'est plus lié à la préférence temporelle des consommateurs, mais à la préférence temporelle des autorités monétaires.

Désormais tout le système des taux relatifs d'intérêt des prêts est hiérarchisé par un prix unique (taux de refinancement de la banque centrale) qui définit le taux de base bancaire de l'ensemble d'une économie nationale. Outre le fait que ce prix de l'argent est partiellement déconnecté des préférences temporelles des acteurs, il centralise tout le jeu des anticipations. Il s'agit de connaître le prix de la monnaie banque centrale. Si toutes les banques anticipent une baisse, alors les entreprises jugent qu'une période d'expansion inflationniste va s'ouvrir et qu'ils ont intérêt à s'endetter pour bénéficier d'un effet de levier.

- Le «big player» transforme le système de règle en un système de commandement. L'institutionnalisation d'un système hiérarchisé rend les entrepreneurs et les banquiers captifs des ordres (commandements) des autorités monétaires qui peuvent exiger des acteurs un type d'action. Le gouverneur de la banque centrale peut, par exemple, souhaiter défendre la valeur de sa monnaie dans une situation où les tensions inflationnistes dominent. Il peut alors obliger les banques quelle que soit leur politique de crédit à déposer une partie de leurs réserves à la banque centrale. Il sanctionne indifféremment les banques qui participent à la crise monétaire et celles qui ont une 
gestion saine de leurs ressources. Il tend alors à ne pas respecter la discipline du marché et à inciter les entrepreneurs à ne pas la respecter puisqu'ils ne seront plus récompensés s'ils anticipent correctement le futur et gèrent sainement leurs affaires.

d) Quelques conséquences sur le débat entre l'école de la libre circulation et l'école de la banque libre

L'introduction d'un «big player» développe par conséquent un processus d'uniformisation des anticipations qui induit une grande instabilité puisque désormais l'erreur ne sera plus isolée, mais collective et plus aucun système de compensation ne pourra jouer. Dans ce contexte, les effets de dominos n'existent que si tous les clients des banques jugent au même moment qu'il existe un risque d'illiquidité. Ils ne s'expliquent que par un processus de convergence des anticipations. Il est peu vraisemblable, cependant, qu'un tel processus existe en économie de marché, car la décentralisation garantit la diversité des anticipations et assure ainsi sa stabilité. L'hypothèse du «big player» explique donc aussi pourquoi un système de banque libre est stable et assuré contre les effets de dominos.

\section{CONCLUSION}

La théorie autrichienne des cycles est donc devenue plurielle et très attentive aux conditions institutionnelles de l'activité des banquiers. Elle a perdu en unité, mais a su renouveler son discours spéculatif (description) et ses prescriptions (morale). 


\section{L'importance des renouvellements théoriques}

L'école autrichienne s'accorde sur l'idée que l'instabilité de l'économie de marché s'explique par la nationalisation de la monnaie mais se divise sur ce qui faisait pourtant le cœur de la théorie autrichienne des cycles puisqu'elle ne s'entend plus sur l'idée que l'origine des cycles s'explique par l'écart entre les crédits accordés par les banques (fiduciary money) et les fonds volontairement épargnés.

Ces divergences ne remettent pas en cause l'unité et l'originalité de sa démarche. Elle est unifiée autour de l'explication de la récurrence des erreurs collectives d'anticipation et propose une théorie endogène des cycles qui met au cœur de son explication l'erreur, le capital et la monnaie. On peut aussi remarquer que la théorie autrichienne des cycles rejoint la théorie post-keynésienne dans sa volonté d'endogénéiser les explications de l'offre de monnaie et de ne plus considérer cette dernière comme une variable exogène. Elle met ainsi l'accent sur le rôle décisif que jouent le banquier et les institutions monétaires (banque centrale ou banque libre) dans l'instabilité des systèmes économiques. Elle se distingue, pour cette raison, des théories exogènes et réelles des cycles où l'erreur d'anticipation est ignorée et où le facteur travail est le seul facteur d'ajustement.

Ces divergences ne sont, cependant, pas sans conséquence, car elles portent sur le cœur de la théorie autrichienne traditionnelle des cycles et sur la nature de la monnaie. Il est juste à notre avis de penser que l'école de la libre circulation est la seule garante de la théorie autrichienne des cycles telle qu'elle s'est développée au début du vingtième siècle. La théorie de la banque libre se présente, au contraire, plus comme une alternative que comme un moyen de perfectionner la théorie existante. Outre ces remarques générales nous pensons que la décentralisation des systèmes de banque libre garantit leur 
stabilité, mais qu'il n'est pas possible de soutenir que les systèmes de réserves fractionnaires qui ont existé ont passé le test du marché. La question reste donc ouverte tant que les Etats n'auront pas libéralisé durablement l'émission de monnaie.

Nous pensons, aussi, que la pratique des réserves fractionnaires explique la mauvaise coordination des projets économiques dans le temps parce qu'elle biaise l'information sur les préférences temporelles véhiculées par les agents. Il n'est pas exclu, pour cette raison, que le test du marché ne sélectionne pas la pratique du coefficient de réserve de $100 \%$ et que la dénationalisation de la monnaie ne garantisse pas contre des baisses artificielles des taux d'intérêt. La mauvaise coordination dans le temps des actions individuelles n'est pas, cependant, générale, car dans un système décentralisé il y a une pluralité de taux d'intérêt. La mauvaise coordination inter-temporelle d'un projet de production explique les faillites individuelles, mais ne provoque pas de crises systémiques. Il ne faut donc pas attendre de la dénationalisation de la monnaie qu'elle assure une parfaite correspondance de tous les projets de production à la demande, mais qu'elle garantisse l'ordre économique contre l'avènement de profondes phases de récession.

\section{Les conséquences de politique économique de la Théorie autrichienne des cycles}

En portant leur attention sur les effets de la nationalisation de la monnaie sur la stabilité des économies de marché, les économistes de l'école autrichienne ont aussi renouvelé leurs prescriptions de politique économique. Ils peuvent soutenir qu'il n'est pas suffisant de constitutionnaliser les politiques monétaires (central circulation school) ${ }^{23}$ pour garantir la stabilité

${ }^{23}$ L'école autrichienne se trouve sur ce point d'accord avec l'analyse proposée par Allais (1993, p.325) ou Friedman (1960). Allais (1993) défend le principe du 
des économies de marché. La constitutionnalisation de la pratique du coefficient de réserve de $100 \%$ rétablit la justesse de l'information véhiculée par les taux d'intérêt sur les préférences temporelles des agents. Elle ne s'attaque, cependant, pas à l'origine de l'instabilité monétaire et économique qui est l'uniformisation des anticipations induite par la nationalisation de la monnaie. Si les économistes autrichiens ont raison, il est juste alors de penser que l'Union monétaire et l'indépendance des banques centrales limitent les erreurs d'anticipation des entrepreneurs et l'apparition de cycle politico-économique, mais renforcent le pouvoir du «big player» et ses effets (la socialisation des pertes ${ }^{24}$, les risques de parasitisme et l'uniformité des anticipations). Ce sont donc des solutions insuffisantes pour lutter efficacement dans le long terme contre l'instabilité des systèmes économiques et monétaires.

coefficient de réserve de $100 \%$ pour faciliter la politique monétaire des gouvernements et suit sur ce point la tradition monétariste qui est favorable au coefficient d'encaisse de $100 \%$ afin de rendre la politique monétaire plus prévisible (Friedman 1960, pp.104-115).

${ }^{24}$ Salin (1991) soutient que la création d'un système monétaire européen autour d'une banque centrale européenne indépendante ne porte pas remède à la caractéristique essentielle des systèmes monétaires modernes, à savoir l'irresponsabilité institutionnelle. 


\section{BIBLIOGRAPHIE}

Allais M. (1993), «Les conditions monétaires d'une économie de marché : des enseignements du passé aux réformes de demain", Revue d'économie politique, vol.103, n³, mai-juin, pp.317-367.

Blaug M. (1981), Histoire de la pensée économique, Economica, Paris.

BLOCK W. (1988), «Fractional-Reserve Banking: An Interdisciplinary Perspective», in Block W. (Ed.), Man Economy, and Liberty: Essays in Honor of Murray N. Rothbard, Llewellyn H. Rockwell, Jr., Auburn, Ala.:Ludwig von Mises Institute, pp.2-31.

BRESCIANI-TURRONI C. (1937), The economics of Inflation, translated by M.E. Sayers, London : George Allen \& Unwin.

Butos W. AND KopPL R.G. (1993), «Hayekian expectations: Theory and Empirical Expectations», Constitutional Political Economy, vol.4, n³, pp.303-330.

CARILli A.M. AND DEMPSTER G.M. (2001), «Expectations in Austrian Business Cycle Theory: An Application of The Prisoner's Dilemma», The Review of Austrian Economics, Vol.14, 4, pp.319330.

Cohran J., Call S.T. and Glahe F. (1999), «Credit Creation of Financial Intermediation?: Fractional-Reserve Banking in a Growing Economy», The Quarterly Journal of Austrian Economics, vol.2, n`3, pp.53-64.

De Mourgues M. (1993), La monnaie. Système financier et théorie monétaire, Economica, Paris.

DOSTALER G. (2001), «La genèse et l'évolution de la théorie des cycles de Hayek», L'actualité économique, Revue d'analyse économique, vol.77, $\mathrm{n}^{\circ} 2$, juin, pp.207-230.

DowD K. (1992) (ed.), The Experience of Free Banking, London: Routledge.

- (1994), «Free Banking», in Boettke P. (Ed.), The Elgar Companion to Austrian Economics, Edward Elgar Publishing, pp.408-413.

FEHL U. (1986), «Spontaneous order and the subjectivity of expectations: a contribution to the Lachmann O'Driscoll Problem», in Kirzner I. (ed.), Subjectivism, Intelligibility and 
Economic Understanding : Essays in Honor of Ludwig von Lachmann on his Eightieth Birthday, New York: NYU Press, pp.72-85.

FRIEDMAN M. (1960), A Program for Monetary Stability, Fordham University Press, New York.

- (1993), "The Plucking Model of Business Fluctuations Revisited», Economic Inquiry, vol.31, April, pp.171-177.

GARRISSON R. (1984), "Time and Money: The Universals of Macroeconomic Theorizing», Journal of Macroeconomics, 6, pp.197-213.

- (1989), «The Austrian Theory of the Business Cycle in the Light of Modern Macroeconomics», The Review of Austrian Economics, 3, pp.3-29.

_ (1996), «Friedman's «Plucking Model», Comment», Economic Inquiry, vol.34, October, pp.799-802.

- (1997), «Austrian Theory of Business Cycles», in Glasner D. (ed.), Business Cycles and Depressions: An Encyclopedia. New York and London: Garland Publishing.

GENTIER A. (2000), «Liberté bancaire ou régulation par une autorité monétaire? Une comparaison de deux systèmes en longue période: - le Massachussetts (1803-1858) — la France (18001870), Journal des économistes et des études humaines, vol. X, numéro 1, mars, pp.110-156.

- (2003) Essai sur les effets de la concurrence et de la réglementation sur le financement du crédit, Publibook, Paris.

GuNNING J.P. (1985), "Causes of unemployement: the Austrian Perspective», History of Political Economy, vol.17, n`2, pp.233-256.

HAYEK F. (1931), Prices and Production; 2 nd edn, revised and enlarged, London: G. Routledge \& Sons, traduction française, Prix et production, coll. Agora (1975). Hayek F. (1932), «A Note on the Development of Doctrine of Forced Saving», Quaterly Journal of Economics, vol.47, pp.123-133.

- (1933), Monetary Theory and the Trade Cycle, traduction de N. Kaldor et H.M. Croomer, Londres, Jonatthan Cape: New York, Harcourt Brace; New York Augustus M. Kelley, 1966.

- (1978), Denationalisation of Money - The Argument Refined: An Analysis of the Theory and Practice of Concurrent Currencies, Second edition, Londres : Institute of Economic Affairs.

- (1952), Sensory Order, Chicago : University of Chicago Press, traduction française, L'ordre sensoriel, CNRS Editions (2001). 
Hoppe H-H. (1994), «How if Fiat Money Possible? Or The Devolution of Money and Credit», The Review of Austrian Economics, 7, (2), pp.49-74.

Hoppe H-H., HÜslmanN J.G., AND Block W. (1998), «Against Fiduciary Media», The Quarterly Journal of Austrian Economics, vol.1, (1), pp.19-50.

HORWITZ S. (1993), «Misreading the «myth» : Rothbard on the theory and history of free banking», in Boettke P.J. and Prychitko D.L. (ed.), The Market Process. Essays in Contemporary Austrian Economics, Edward Elgar, pp.166-176.

HUERTA DE SOTO J. (1994), «Banque centrale ou banque libre : le débat théorique sur les reserves fractionnaires», Journal des économistes et des études humaines, vol.V, numéro $2 / 3$, juinseptembre, pp.379-391.

- (1995), «A Critical Analysis of Central Banks and FractionalReserve Free Banking from the Austrian Perpspective», The Review of Austrian Economics, vol.8, (2), pp.25-38.

- (1996), «New Light on the Prehistory of the Theory of Banking and the School of Salamanca», The Review of Austrian Economics, vol.9, n²,pp.59-81.

- (1997), «The Spanish Roots of the Austrian School», Austrians Economics Newsletter, summer.

- (1998a), Dinero, Credito Bancario y Ciclos Económicos. Madrid: Union Editorial.

- (1998b), "A Critical Note on Fractional-Reserve Free Banking», The Quarterly Journal of Austrian Economics, vol.1, (4), pp.25-49.

HugHes A.M.(1997), «The Recession of 1990: An Austrian Explanation», The Review of Austrian Economics, 10, (1), pp.107-123.

HÜLSMANN J.G. (1996), «Free Banking and the Free Bankers», The Review of Austrian Economics, vol.9, n¹, pp.3-53.

HÜLSMANN J.G. (1998), «A General Theory of Error Cycles», The Quarterly Journal of Austrian Economics, vol.1; ${ }^{\circ} 4$, pp.1-24.

- (1998b), «Free Banking and Fractional Reserves : Reply to Pascal Salin», The Quaterly Journal of Austrian Economics, vol.1, n³, pp.67-71.

- (2000a), «Théorie et théoriciens de la banque libre commentaire de Le Maux», Journal des économistes et des études humaines, vol.10, $\mathrm{n}^{\circ} 1$, mars, pp.157-167. 
- (2000b), «Banks Cannot Create Money», The Independent Review, vol.5, $\mathrm{n}^{\circ} 1$, summer, pp.101-110.

- (2000c), «Product Differenciation and Fractional-Reserve Banking», a previous version of this paper has been presented as «Government, Fractional-Reserve, and Financial Crise», at the Twenty-fith Annual Conference of the association of Private Enterprise Education, 2-4 April 2000, Las Vegas, Nevada.

KEELER J.P. (2001), «Empirical Evidence on the Austrian Business Cycle Theory», The Review of Austrian Economics, 14, (4), pp.331-351.

Koppl R.G. AND Yeager L.B. (1992), «Big Players and Herding in Asset Markets: The Case of the Russian Ruble», Explorations in Economic History, vol.38, pp.367-383.

LACHMANN L. (1978), Capital and its Structure, Sheed Andrews and McMeel, INC, Kansas City, reed. 1955.

LeIJONHUfVUd A. (1986), «Real and Monetary Factors in Business Fluctuations», Cato Journal, 6, (2), pp.409-420.

Le MauX L. (2000), «Ludwig von Mises, Théoricien de la Banque libre : réponse à J.G. Hülsmann», Journal des économistes et des études humaines, vol.10, n¹, mars, pp.169-178.

Le Roux P. and M. LeVIN (1998), "The Capital Structure and the Business Cycle : Some Test of the Validity of the Austrian Business Cycle in South Africa», Journal for Studies in Economics and Econometrics, 22, (3), pp.91-109.

Mises L. $(1912,1980)$, «The Theory of Money and Credit, Indianapolis: Liberty Fund.

- (1985), L'action Humaine, coll. Libre échange, PUF, Paris, traduit de l'américain Human Action. A Treatise on Economics, ed.1966, Henry Regnery Company, Chicago.

NAtAF P. (1997), "Le secret de la liberté des banques et de la monnaie», dans Madelin A. (Ed.), Aux Sources du modèle libéral français, Perrin, pp.189-209.

O'Driscoll G. AND Rizzo M. (1985), The Economics of Time and Ignorance, Oxford: Basil Blackwell.

RobBins L. (1934), The Great Depression, London: Macmillan.

RothBARD M. (1970), Man, Economy, and State: A Treatise on Economic Principles. Los Angeles: Nash Publishing.

- (1975), America's Great Depression, Kansas City: Sheed and Ward. 
- (1983), The Mystery of Banking. New York: Richardson and Snyder.

- (1988), «The Myth of Free Banking in Scotland», The Review of Austrian Economics, vol.2, pp.229-245.

SALERNO J. (1983), «Gold Standards: True and False», Cato Journal, vol.3, spring, pp.239-237.

- (1993), «Mises and Hayek Dehomogenized», The Review of Austrian Economics, 6, (2), pp.113-146.

SALERNO J.T. (1994), «Ludwig von Mises's Monetary Theory in Light of Modern Monetary Though», The Review of Austrian Economics, vol.8, n ${ }^{\circ}$, pp.71-115.

SALIN P. (1991), La vérité sur la monnaie, édition Odile Jacob, Paris.

- (1991b), «Quelles monnaies pour le marché unique européen», Revue d'économie politique, 101, (1), janv.-fév., pp.110-126.

- (1998), «Free Banking and Fractional Reserves: A Comment on Guido Hülsmann», The Quarterly Journal of Austrian Economics, vol. 1, n³, Fall, pp.61-66.

- (2001), «In Defence of Fractional Monetary Reserves», A provisional version of this paper has been presented at the Austrian Scholars Conference, Mises Institute, Auburn, March, 30-31, 2001.

SeChrest L.J. (1997), «Austrian and Monetarist Business Cycle Theories: Substitutes or Complements?», in Boettke P.K and Horwitz S. (Ed.), Advances in Austrian Economics, vol.4, Symposium on Austrians Macroeconomics, JAI Press, INC, pp.7-32.

SELGIN G. (1988), The Theory of Free Banking : Money Supply Under Comptetive Note Issue, Totowa, N.J.: Rowman and Littlefield traduction française (1991), La théorie de la banque libre, Les Belles Lettres, coll. Laissez faire.

SelgiN G. AND White L. (1996), «In Defense of Fiduciairy Media; or, We Are Not Devo(lutionists), We Are Misesians!», The Review of Austrian Economics, vol.9, pp.83-107.

SElgin G. (2000), «Should we let banks Create Money?», The Independent Review, v.V., $\mathrm{n}^{\circ} 1$ Summer, pp.93-100.

SKOUSEN M. (1988), «Saving the Depression : A New Look at World War II», The Review of Austrian Economics, 2, pp.211-226. 
- (1994), «The Great Depression», in Boettke P.J. (Ed.), The Elgar Comparion to Austrian Economics, Edward Elgar Publishing, Cheltenham UK, Northampton, MA, USA, pp.431-439.

WAINHOUSE C. (1984), «Empirical Evidence for Hayek's Theory of Economic Fluctuations», in Siegel B. (Ed.) Money in Crisis. San Francisco: Pacific Institute for Public Policy Research, pp.37-71.

White L. H. (1984), Free Banking in Britain, Experience, and Debate, 1800-1845, Cambridge/New York: Cambridge University Press.

- (1989), Competition and Currency. New York: New York University Press.

- $\quad$ (1995), Free Banking in Britain, 2. ${ }^{\circ}$ Ed., London: Institute for Economic Affairs.

- (1999), The Theory of Monetary Institutions, Oxford: Blacwell.

YEAGER L.B. (1986), «The Significance of Monetary Disequilibrium», The Cato Journal, vol.6, n², fall, pp.369-399.

- (2001), "The peril of Base Money», The Review of Austrian Economics, vol.14, Number 4, pp.251-266. 